\title{
A positive feedback loop formed by NGFR and FOXP3 contributes to the resistance of non-small cell lung cancer to icotinib
}

\author{
Jun Huang ${ }^{1 \#}$, Qiuhua Yu ${ }^{1 \#}$, Yanjuan Zhou ${ }^{2}$, Ying $\mathrm{Chu}^{3}$, Feng Jiang ${ }^{1}$, Xiaobo Zhu ${ }^{1}$, Junjie Zhang ${ }^{1}$, \\ Qiang Wang ${ }^{1}$
}

${ }^{1}$ Department of Thoracic-Cardiology, ${ }^{2}$ Department of Respiratory, ${ }^{3}$ Department of Science and Education Division, Wujin Hospital Affiliated with Jiangsu University, The Wujin Clinical College of Xuzhou Medical University, Changzhou 213017, China

Contributions: (I) Conception and design: Q Wang; (II) Administrative support: J Huang, Q Yu; (III) Provision of study materials or patients: Y Zhou; (IV) Collection and assembly of data: Y Chu, F Jiang; (V) Data analysis and interpretation: X Zhu, J Zhang; (VI) Manuscript writing: All authors; (VII) Final approval of manuscript: All authors.

\#These authors contributed equally to this work.

Correspondence to: Qiang Wang. Professor, Department of Thoracic-Cardiology, Affiliated Wujin Hospital of Jiangsu University, 2nd Yongning North Road, Tianning District, Changzhou 213017, China. Email: wq66806@163.com.

\begin{abstract}
Background: The study was aimed to investigate the mechanisms causing acquired chemoresistance to icotinib, an epidermal growth factor receptor tyrosine kinase inhibitor (EGFR-TKI), in non-small cell lung cancer (NSCLC).

Methods: Three wildtype NSCLC cell lines were used to produce icotinib-resistant (IR) cell lines. Realtime PCR and western blot assays were used to detect the mRNA and protein levels of nerve growth factor receptor (NGFR) and forkhead box P3 (FOXP3). MTT assay was used to detect the viability of cells. Luciferase activity and chromatin immunoprecipitation (ChIP) assays were used to detect the transactivation activity of FOXP3.

Results: NGFR and FOXP3 were dramatically increased in three IR NSCLC cell lines, and both proteins were required for induction of icotinib resistance. NGFR-induced icotinib resistance was partially related to activation of AKT, a well-known chemoresistance inducer in many tumor types. Activated AKT could induce the expression of FOXP3 which further induce icotinib through transactivating NGFR expression by binding to its promoter. In addition, the inducing of FOXP3 could also induce icotinib resistance solely.

Conclusions: NGFR, AKT and FOXP3 form a positive feedback loop, by which the abilities of NGFR and FOXP3 on inducing icotinib resistance are further enhanced. We believe that NGFR and FOXP3 might be novel therapeutic targets in NSCLC.
\end{abstract}

Keywords: Non-small cell lung cancer (NSCLC); icotinib; nerve growth factor receptor (NGFR); forkhead box P3 (FOXP3); AKT

Submitted Jun 26, 2019. Accepted for publication Nov 29, 2019.

doi: $10.21037 /$ tcr.2019.12.60

View this article at: http://dx.doi.org/10.21037/tcr.2019.12.60

\section{Introduction}

Non-small cell lung cancer (NSCLC) is the leading cause of cancer-related death (1). Eighty to eighty-five percent of lung cancer cases are NSCLC (1). Because the lack of cancer-specific symptoms, the majority of NSCLC cases are diagnosed at advanced stage, which always results in poor prognosis of patients with NSCLC (2).

Because $10-35 \%$ NSCLCs are driven by epidermal growth factor receptor (EGFR) mutations, EGFR has emerged as the most successive target for NSCLC therapy (3). However, only a limited subset of patients harboring EGFR mutations benefit from the EGFR tyrosine kinase inhibitors (EGFR-TKIs) therapy, the majority of NSCLC patients 
are EGFR overexpression but not EGFR mutations (4). Intrinsic resistance in patients without EGFR mutations and acquired resistance after the initial response to TKIs are serious clinical issues. Uncovering the mechanisms by which chemoresistance is induced is beneficial for the improvement of the efficacy of TKIs in NSCLC.

Icotinib, a novel and the third listed EGFR-TKIs, has been approved as the first-line therapy in patients with advanced NSCLC by China Food and Drug Administration (5). Although it shows a good anti-tumor efficacy on NSCLC (6), especially in the advanced NSCLC (7), the resistance to icotinib is still observed in clinic (8). Here, in three icotinibresistant (IR) NSCLC cell lines, nerve growth factor receptor (NGFR) and forkhead box P3 (FOXP3) were identified to be novel inducer of icotinib resistance, and a positive feedback loop formed by the two proteins amplified their effects on inducing icotinib resistance.

\section{Methods}

\section{Cell culture and establishment of IR NSCLC cell lines}

Three wildtype NSCLC cell lines, A549, NCI-H661 and PC9, were cultured in RPMI-1640 medium (Gibco, USA) supplemented with $10 \%$ fetal bovine serum, penicillin $(100 \mathrm{U} / \mathrm{mL})$, and streptomycin $(100 \mathrm{mg} / \mathrm{mL})$ at $37^{\circ} \mathrm{C}$ under $5 \% \mathrm{CO}_{2}$. To establish three IR NSCLC cell lines, A549, HCI-H661 and PC9 were cultured with incrementing doses of icotinib (Selleck, USA) starting from $1 \mu \mathrm{M}$ for 2 weeks, $3 \mu \mathrm{M}$ for 2 weeks, $5 \mu \mathrm{M}$ for 4 weeks, $8 \mu \mathrm{M}$ for 6 weeks and $10 \mu \mathrm{M}$ for 10 weeks.

\section{Cell treatment}

AT7867 (Selleck) was prepared in 5\% dimethyl sulfoxide to obtain a stock solution of $20 \mathrm{mM}$, and $15 \mu \mathrm{M}$ AT7867 were used to treat cells. Lipofectamine 2000 (Thermo Fisher, USA) was used to transfect luciferase reporter plasmid containing NGFR promoter or plasmid encoding human FOXP3 according to the manufacturer's protocol. Lipofectamine RNAiMAX (Thermo Fisher) was used to transfect specific siRNAs of NGFR/FOXP3 (Santa Cruz, USA) and control siRNA (Santa Cruz) according to the manufacturer's protocol.

\section{MTT assay}

MTT colorimetric assay kit (Promega, USA) was used to detect cell viability as previously described (9). Briefly, cells were plated in 96-well plates at a density of 5,000 cells, followed by culturing for 24 hours. The next day, cells were transfected with plasmids encoding NGFR/FOXP3 siRNAs or human FOXP3, or treated with icotinib/ AT7867 for indicated times. MTT regent was added, and then cells were incubated at $37^{\circ} \mathrm{C}$. After 4 hours, formazan crystals were solubilized, and absorbance was measured at a wavelength of $570 \mathrm{~nm}$.

\section{Luciferase activity assay}

The detailed method of luciferase activity assay had been described previously (10). Briefly, cells were transfected with promoter-reporter plasmid that contain NGFR promoter. Relative light unit values were normalized to the $\beta$-galactosidase signal. One microgram of promoterreporter plasmid and pRSV $\beta$-galactosidase plasmid was used for all transfections.

\section{TaqMan-based real-time PCR}

The mRNA levels of NGFR, AKT and FOXP3 were detected by TaqMan-based real-time PCR as previously described (11). Briefly, the RNeasy Mini Kit (Qiagen, USA) was used to isolate total RNA and reverse transcription was used to synthesize first-strand cDNA using the SuperScript II First-Strand Synthesis System for RT-PCR (Invitrogen, USA). mRNA contents of NGFR/ AKT/FOXP3 were normalized to the housekeeping gene $\beta$-actin. The primers and probes for detecting $\beta$-actin, FOXP3, NGFR and AKT were obtained from Fisher Scientific.

\section{Western blot}

Protein levels were detected by western blot as previously described (11). Briefly, RIPA Lysis and Extraction Buffer (Thermo Fisher) was used to isolated total protein. Isolated proteins $(10-50 \mu \mathrm{g})$ were separated on $10-15 \%$ SDS-PAGE gels, followed by transferring of separated proteins to PVDF membranes. Five percent non-fat milk was used to block PVDF membrane. Anti-NGFR (Abcam, USA), antiPARP (Abcam), anti-cleaved PARP (Abcam), anti-FOXP3 (Abcam), anti-AKT (Abcam), anti-p-AKT(S473) (Abcam) and anti- $\beta$-actin (Santa Cruz) were used for the specific primary antibodies. 


\section{Chromatin immunoprecipitation (ChIP)}

The interaction between FOXP3 and NGFR promoter was determined by ChIP as previously described (12). Briefly, antiFOXP3 antibody (Abcam) was used for immunoprecipitating the DNA fragments that interacted with FOXP3, followed by PCR and agarose gel electrophoresis. The PCR of the NGFR promoter sequence was formed using 40 cycles as follows: $95^{\circ} \mathrm{C}$ for 15 seconds, $65^{\circ} \mathrm{C}$ for 40 seconds. The PCR products were analyzed on $1.5 \%$ agarose gels.

\section{Statistical analysis}

All the data shown are the results of at least three independent experiments and are expressed as means \pm standard error of the mean (SEM). The differences between groups were compared using Student's $t$-test. Differences were considered significant at confidence levels of $\mathrm{P}<0.05$, $\mathrm{P}<0.01$ and $\mathrm{P}<0.001$, as indicated.

\section{Results}

\section{NGFR is required for induction of icotinib resistance in NSCLC cells}

A549, NCI-H661 and PC9 cell lines were used to produce three IR cell lines (A549-IR, NCI-H661-IR and PC9-IR) which successfully grew in medium with $10 \mu \mathrm{M}$ icotinib. Compared with wildtype cell lines, mRNA and protein levels of NGFR were significantly increased in three IR NSCLC cell lines (Figure 1A,B). Ten $\mu \mathrm{M}$ icotinib induced cleaved PARP, a marker of apoptosis, in three IR NSCLC cell lines with the knockdown of NGFR via its specific siRNA and in three wildtype cell lines, but not in the IR cell lines expressing control siRNA (Figure 1C). Ten $\mu \mathrm{M}$ icotinib also reduced the viabilities of three IR cell lines expressing NGFR siRNA and three wildtype cell lines, but did not reduce the viabilities of three IR cell lines expressing control siRNA (Figure 1D). These results indicate an important role of NGFR on inducing icotinib resistance by suppressing apoptosis and maintaining cell viability.

\section{FOXP3 is also required for induction of icotinib resistance in NSCLC cells}

Compared with wildtype cell lines, the expression of FOXP3 was significantly increased in three IR NSCLC cell lines (Figure 2A,B), and siRNA-mediated FOXP3 silencing recovered the abilities of icotinib $(10 \mu \mathrm{M})$ on inducing apoptosis and reducing cell viability in these IR cell lines, suggesting an important role of FOXP3 on inducing icotinib resistance in NSCLC cells by inhibiting apoptosis and maintaining cell viability (Figure $2 C, D$ ). Moreover, $10 \mu \mathrm{M}$ icotinib treatment could not reduce the viabilities of three wildtype NSCLC cell lines with FOXP3 overexpression, suggesting that the inducing of FOXP3 can induce icotinib resistance solely (Figure 2E).

\section{FOXP3 induces NGFR transcription by binding to its promoter}

First, the knockdown of FOXP3 reduced mRNA and protein levels of NGFR in three IR cell lines (Figure $3 A, B)$, but FOXP3 overexpression would increase NGFR expression (Figure 3C,D), suggesting that FOXP3 can positively regulate NGFR expression. Next, a NGFR luciferase (NGFR-luc) reporter and a plasmid encoding human FOXP3 were co-transfected into three wildtype NSCLC cell lines for 48 hours. As shown in Figure $3 E$, FOXP3 overexpression enhanced the luciferase activity of NGFR-luc, suggesting an important role of FOXP3 on transactivating NGFR expression (Figure 3E). At last, the binding of FOXP3 to NGFR promoter in three wildtype NSCLC cell lines was determined by ChIP assay, suggesting that NGFR is a bona fide transcriptional target of FOXP3 and its transcription can be induced by FOXP3 binding to its promoter (Figure $3 F$ ). ChIP assay further identified more binding in three IR cells lines than in wildtype cell lines (Figure 3G), partially explaining why NGFR was increased in three IR cell lines.

\section{NGFR induces the expression of FOXP3 by activating AKT}

As shown in Figure 4A,B, although mRNA and total protein levels of AKT in three IR NSCLC cell lines were similar as that in wildtype cell lines, the protein levels of p-AKT(S473) in IR cell lines were higher than that in wildtype cell lines, suggesting that AKT was activated in IR NSCLC cells. Inhibition of AKT activity by $15 \mu \mathrm{M}$ AT7867 significantly reduced the viabilities of three IR cell lines treated with $10 \mu \mathrm{M}$ icotinib, suggesting a critical role of activated AKT on maintaining the viability of IR NSCLC cells (Figure 4C). Inhibition of AKT activity via AT7867 resulted in reduction of mRNA and protein levels of FOXP3 in three IR NSCLC cell lines, suggesting that activated AKT is required for inducing FOXP3 expression (Figure 4D,E). Finally, knockdown of NGFR via its specific siRNA reduced the 

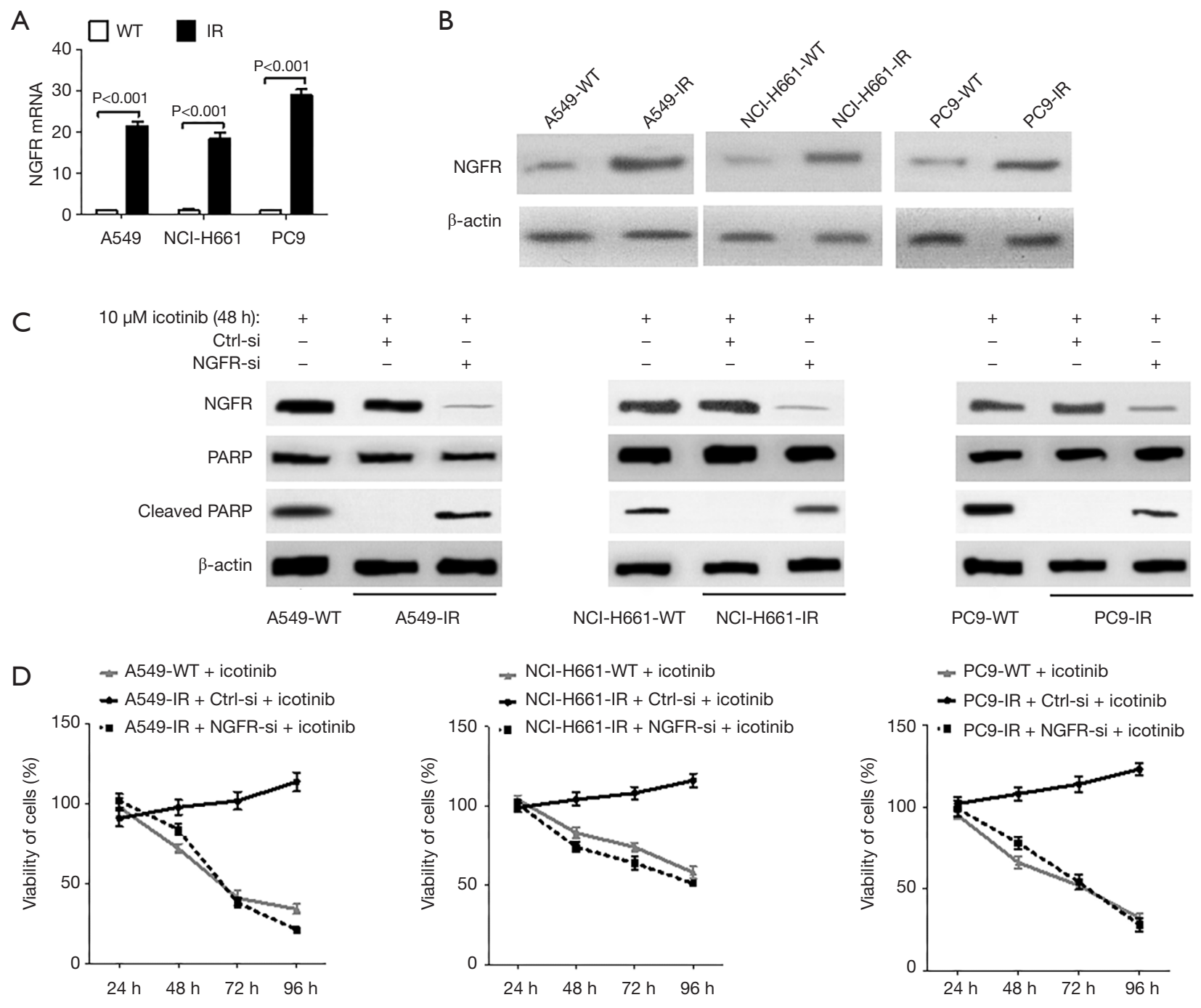

Figure 1 Detection the effect of NGFR on inducing icotinib resistance in NSCLC cell lines. Real-time PCR (A) and western blot (B) detection of mRNA and protein levels of NGFR in three IR NSCLC cell lines (A549-IR, NCI-H661-IR, PC9-IR) and WT cell lines (A549WT, HCI-H661-WT, PC9-WT); WT and IR NSCLC cell lines were previously transfected with NGFR siRNA (NGFR-si) or control siRNA (Ctrl-si) for 6 hours, followed by icotinib treatment $(10 \mu \mathrm{M})$ for indicated times; western blot detection of indicated proteins (C) and MTT detection of cell viability (D). NGFR, nerve growth factor receptor; NSCLC, non-small cell lung cancer; IR, icotinib-resistant; WT, wildtype.

protein levels of p-AKT(S473), but not total AKT, in three IR cell lines, suggesting that NGFR expression is required for activation of AKT (Figure $4 F$ ). Taken together, these data suggest that NGFR can induce FOXP3 expression by activating AKT.

\section{Discussion}

In this study, our data demonstrate new roles of NGFR and FOXP3 on inducing icotinib resistance in NSCLC cells and further uncover a positive feedback loop formed by NGFR, AKT and FOXP3 (Figure 4G). This feedback loop undoubtedly can enlarge the effects of NGFR and FOXP3 on inducing icotinib resistance (Figure $4 G$ ). To our knowledge, these data have rarely been reported.

NGFR, a $75 \mathrm{kD}$ single-transmembrane protein, widely expresses in the central and peripheral nervous system (13). It does not have kinase activity but often partners with other 

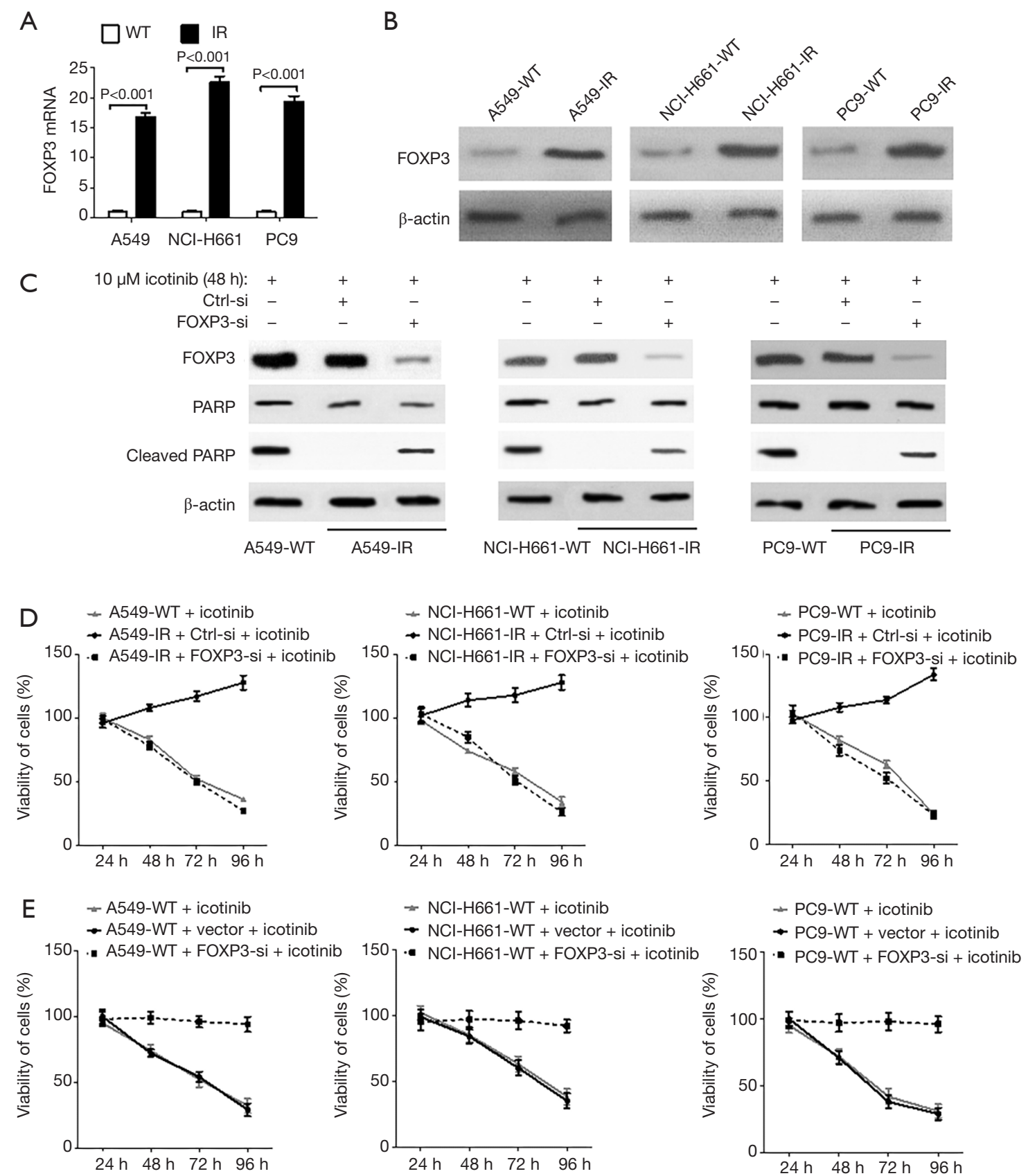

Figure 2 Detection the effect of FOXP3 on inducing icotinib resistance in NSCLC cell lines. Real-time PCR (A) and western blot (B) detection of mRNA and protein levels of FOXP3 in three IR NSCLC cell lines and WT cell lines; (C,D) WT and IR NSCLC cell lines were previously transfected with FOXP3 siRNA (FOXP3-si) or control siRNA (Ctrl-si) for 6 hours, followed by icotinib treatment (10 $\mu M)$ for indicated times. Western blot detection of indicated proteins (C) and MTT detection of cell viability (D); (E) WT and IR NSCLC cell lines were previously transfected with plasmid encoding human FOXP3 or control vector for 6 hours, followed by icotinib treatment $(10 \mu \mathrm{M})$. MTT detection of cell viability at indicated times. FOXP3, forkhead box P3; NSCLC, non-small cell lung cancer; IR, icotinibresistant; WT, wildtype. 

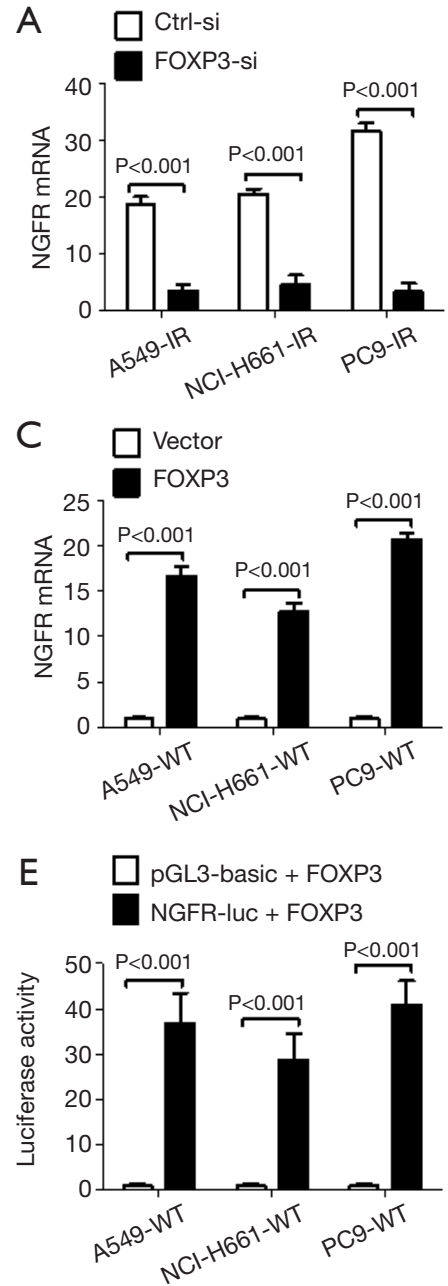
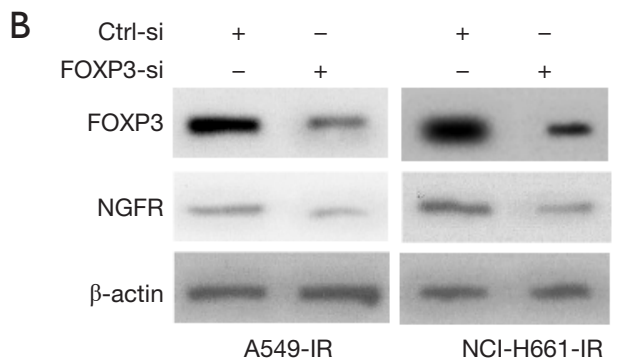

$\mathrm{NCl}-\mathrm{H} 661-\mathrm{IR}$
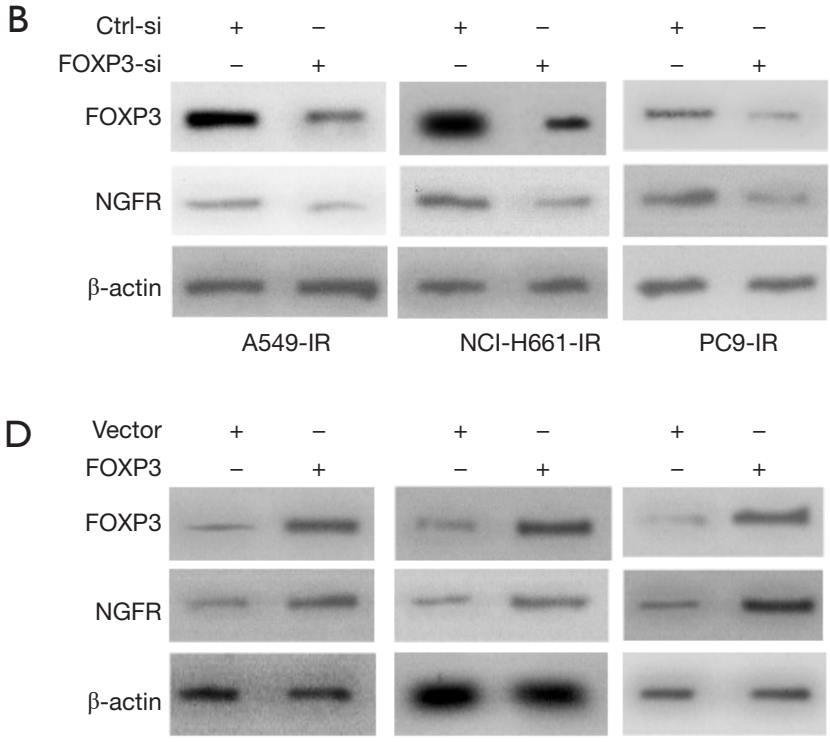

A549-WT

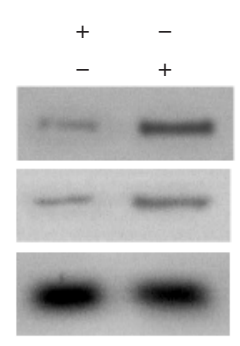

NCl-H661-WT

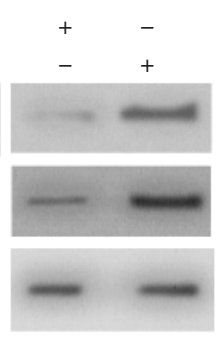

PC9-WT
$\mathrm{F}$

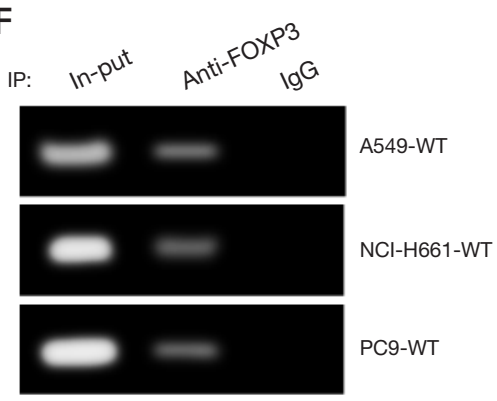

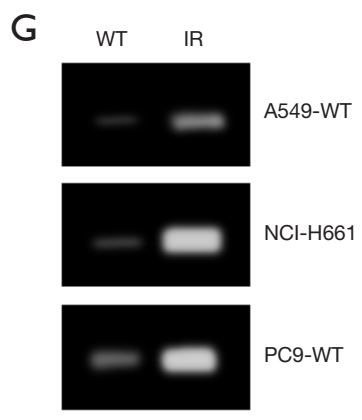

Figure 3 FOXP3 promotes NGFR expression by inducing its transcription. (A,B) Three IR NSCLC cell lines were transfected with FOXP3 siRNA (FOXP3-si) or control siRNA (Ctrl-si) for 48 hours, followed by detection of NGFR mRNA by real-time PCR (A) and its protein by western blot (B); (C,D) three WT NSCLC cell lines were transfected with plasmid encoding human FOXP3 or control vector for 48 hours, followed by detection of NGFR mRNA by real-time PCR (C) and its protein by western blot (D); (E) three WT NSCLC cell lines were cotransfected with plasmid encoding human FOXP3 and luciferase reporter plasmid containing NGFR promoter (NGFR-luc) reporter vector (pGL3-basic) for 48 hours, followed by detection of luciferase activity; (F) detection the binding of FOXP3 to NGFR promoter by ChIP. An anti-FOXP3 antibody was used for immunoprecipitating FOXP3-binding DNA fragments, followed by PCR to amply NGFR promoter and ethidium bromide-stained agarose gel electrophoresis detection of PCR productor; (G) detection the bindings of FOXP3 to NGFR promoter in WT and IR NSCLC cell lines by ChIP. FOXP3, forkhead box P3; NGFR, nerve growth factor receptor; NSCLC, non-small cell lung cancer; NGFR-luc, NGFR luciferase; ChIP, chromatin immunoprecipitation; IR, icotinib-resistant; WT, wildtype.

receptors, e.g., TrkA, to be involved in regulating multitude of processes during neurogenesis (13). The roles of NGFR on tumor are tumor cells-type dependent. For example, activation of NGFR inhibits apoptosis and increased survival of schwannoma and breast cancer cells $(14,15)$, and it is also required for promoting migration and invasion in many metastatic tumors (16). But NGFR is reported to suppress tumor growth in prostate and bladder cancers $(17,18)$. The binding of SHC1 to NGFR can activate PI3K/AKT pathway (19). Abnormal activation of PI3K/AKT pathway has been frequently observed in many types of tumor cells, which supports survival of tumor cells and is required for 
A

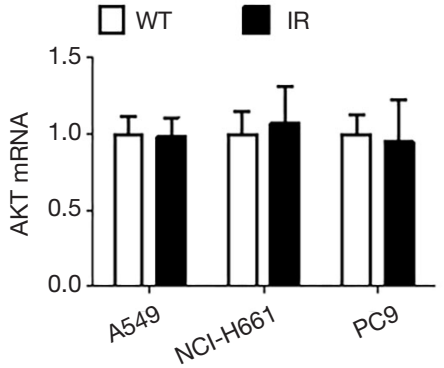

C
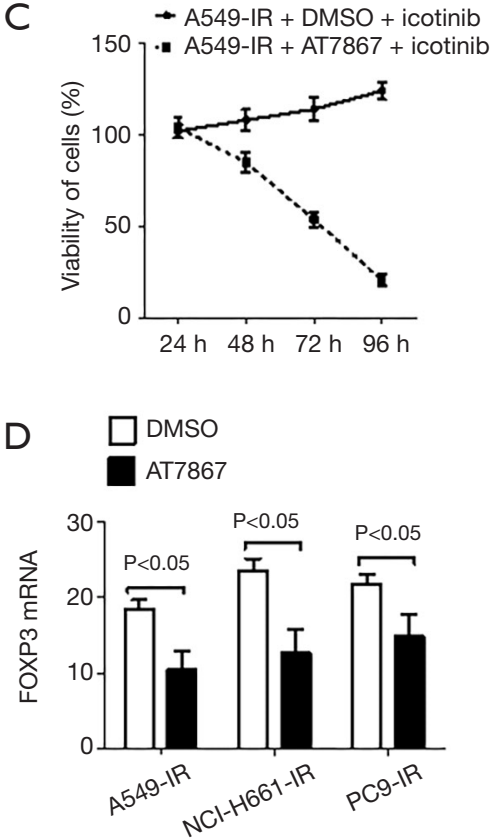

$\mathrm{F}$

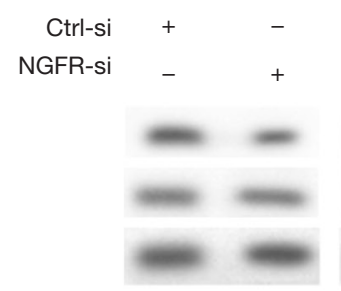

A549-IR
B
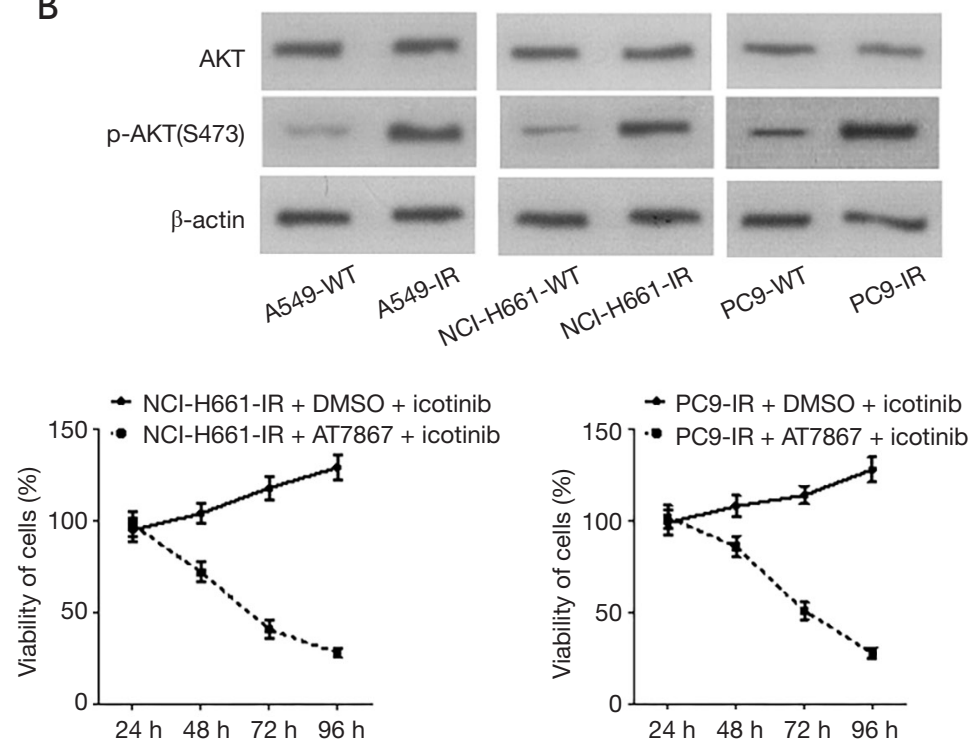

$E$

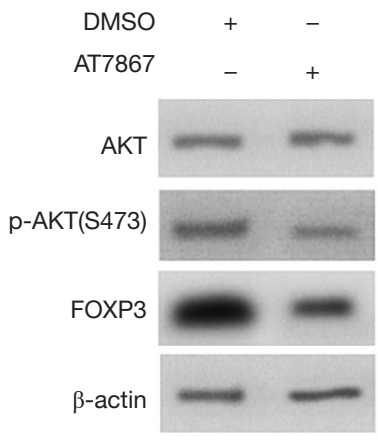

A549-IR

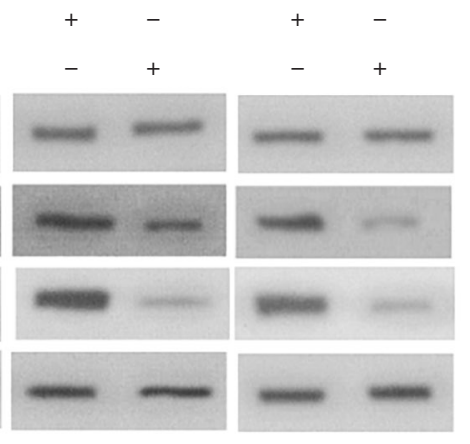

$\mathrm{NCl}-\mathrm{H} 661-\mathrm{IR}$

PC9-IR

G

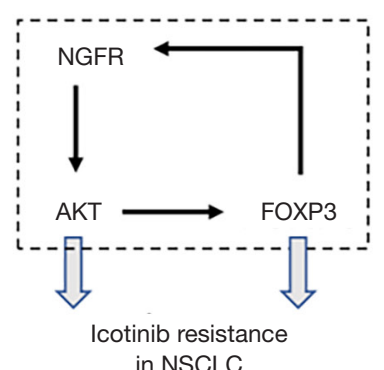

Figure 4 NGFR-activated AKT induces FOXP3 expression. (A) Real-time PCR detection of AKT mRNA in three WT and IR NSCLC cell lines; (B) western blot detection of indicated proteins in three WT and IR NSCLC cell lines; (C) MTT detection of the viability of three IR NSCLC cells co-treated with AT7867 $(15 \mu M)$ and icotinib $(10 \mu M)$ for indicated times; (D) real-time PCR detection of FOXP3 mRNA in three IR NSCLC cell lines treated with AT7867 $(15 \mu \mathrm{M})$ or DMSO for 48 hours; (E) western blot detection of indicated proteins in three IR NSCLC cell lines treated with AT7867 $(15 \mu \mathrm{M})$ or DMSO for 48 hours; (F) western blot detection of indicated proteins in three IR NSCLC cell lines transfected with NGFR siRNA (NGFR-si) or control siRNA (Ctrl-si) for 48 hours; (G) a model for the role of the positive feedback loop formed by NGFR, AKT and FOXP3 in inducing icotinib resistance in NSCLC cells. FOXP3, forkhead box P3; NGFR, nerve growth factor receptor; NSCLC, non-small cell lung cancer; IR, icotinib-resistant; WT, wildtype. 
induction of chemoresistance (20). Although the effects of NGFR on induction of chemoresistance are rarely reported, it is rational to believe that NGFR-induced chemoresistance might be dependent on activating PI3K/AKT pathway. Our data confirmed that, at least in NSCLC cells, NGFR activates AKT to induce the resistance to icotinib. NGFR is also required for induction of cancer stem cells (CSCs) (21). CSCs possess strong chemoresistance capacity by expressing drug-transporter proteins, which lead to high resistance to chemotherapeutic agents (22). We believe that NGFR might also induce chemoresistance by inducing CSCs.

FOXP3 is a critical transcription factor for induction of $\mathrm{CD} 4^{+} \mathrm{CD} 25^{+} \mathrm{T}$ regulatory cells (Treg) $(23,24)$. Treg cells serve an immunosuppressive function in the tumor microenvironment and contribute to the escape of tumor cells from the immune system and contribute to the progression of many tumor types (including NSCLC) (25-27). The role of FOXP3 on promoting NSCLC through inducing Treg development is clear $(26,27)$, but the role of FOXP3 expressed by tumor cells remains controversial. FOXP3 expressed in human tumor samples (including NSCLC) is related to metastatic spread, lymph node metastasis and TNM stage, but it is regarded as a tumor suppressor in vitro studies (28-30). Although FOXP3 is reported to induce chemoresistance through promoting Treg development $(23,24)$, the effect of FOXP3 expressed by NSCLC cells on inducing chemoresistance is rarely reported. FOXP3 has many transcriptional target genes including many oncogenes (31). Thus, it is rational to believe that FOXP3induced expression of many oncogenes should partially contribute to its effect on inducing chemoresistance.

Taken together, our data demonstrate that NGFR and FOXP3 are two critical factors causing the resistance to icotinib in NSCLC cells. We believe that both proteins should have great potential to be new therapeutic targets in NSCLC.

\section{Acknowledgments}

Funding: This study was supported by grant from the Natural Science Foundation of Jiangsu Province, China (BK20151175) and from the Wujin District, Changzhou City, Jiangsu Province Science and Technology Support Project Funding (WS201701, WS201803).

\section{Footnote}

Conflicts of Interest: All authors have completed the ICMJE uniform disclosure form (available at http://dx.doi. org/10.21037/tcr.2019.12.60). The authors have no conflicts of interest to declare.

Ethical Statement: The authors are accountable for all aspects of the work in ensuring that questions related to the accuracy or integrity of any part of the work are appropriately investigated and resolved. The study was approved by the Human Ethics Committee at the Wujin Hospital Affiliated with Jiangsu University (No.202005).

Open Access Statement: This is an Open Access article distributed in accordance with the Creative Commons Attribution-NonCommercial-NoDerivs 4.0 International License (CC BY-NC-ND 4.0), which permits the noncommercial replication and distribution of the article with the strict proviso that no changes or edits are made and the original work is properly cited (including links to both the formal publication through the relevant DOI and the license). See: https://creativecommons.org/licenses/by-nc-nd/4.0/.

\section{References}

1. Park S, Langley E, Sun JM, et al. Low EGFR/MET ratio is associated with resistance to EGFR inhibitors in nonsmall cell lung cancer. Oncotarget 2015;6:30929-38.

2. Spiro SG, Tanner NT, Silvestri GA, et al. Lung cancer: progress in diagnosis, staging and therapy. Respirology 2010;15:44-50.

3. Dong L, Lei D, Zhang H. Clinical strategies for acquired epidermal growth factor receptor tyrosine kinase inhibitor resistance in non-small-cell lung cancer patients. Oncotarget 2017;8:64600-6.

4. Sharma SV, Bell DW, Settleman J, et al. Epidermal growth factor receptor mutations in lung cancer. Nat Rev Cancer 2007;7:169-81.

5. Shi Y, Sun Y, Yu J, et al. China experts consensus on the diagnosis and treatment of advanced stage primary lung cancer (2016 version). Asia Pac J Clin Oncol 2017;13:87-103.

6. Xue ZX, Wen WX, Zhuang Y, et al. Comparison of the efficacy of icotinib in patients with non-small-cell lung cancer according to the type of epidermal growth factor receptor mutation. Mol Clin Oncol 2016;5:265-8.

7. Shi $Y$, Zhang $L$, Liu $X$, et al. Icotinib versus gefitinib in previously treated advanced non-small-cell lung cancer (ICOGEN): a randomised, double-blind phase 3 noninferiority trial. Lancet Oncol 2013;14:953-61.

8. Zheng H, Wang Q, Shi H, et al. Favorable response to 
icotinib in a lung cancer patient with a special mutation at exon 19 of epidermal growth factor receptor. Thorac Cancer 2014;5:358-61.

9. Xiao W, Gao Z, Duan Y, et al. Notch signaling plays a crucial role in cancer stem-like cells maintaining stemness and mediating chemotaxis in renal cell carcinoma. J Exp Clin Cancer Res 2017;36:41.

10. Chen Y, Zeng Q, Liu X, et al. LINE-1 ORF-1p enhances the transcription factor activity of pregnenolone $\mathrm{X}$ receptor and promotes sorafenib resistance in hepatocellular carcinoma cells. Cancer Manag Res 2018;10:4421-38.

11. Zheng M, Xu H, Liao XH, et al. Inhibition of the prolyl isomerase Pin1 enhances the ability of sorafenib to induce cell death and inhibit tumor growth in hepatocellular carcinoma. Oncotarget 2017;8:29771-84.

12. Yang TY, Teng CJ, Lin TC, et al. Transcriptional repression of Aurora-A gene by wild-type p53 through directly binding to its promoter with histone deacetylase 1 and $\mathrm{mSin} 3 \mathrm{a}$. Int J Cancer 2018;142:92-108.

13. Barker PA. P75NTR is positively promiscuous: novel partners and new insights. Neuron 2004;42:529-33.

14. Ahmad I, Yue WY, Fernando A, et al. P75NTR is highly expressed in vestibular schwannomas and promotes cell survival by activating nuclear transcription factor kappaB. Glia 2014;62:1699-712.

15. Descamps S, Toillon RA, Adriaenssens E, et al. Nerve growth factor stimulates proliferation and survival of human breast cancer cells through two distinct signaling pathways. J Biol Chem 2001;276:17864-70.

16. Civenni G, Walter A, Kobert N, et al. Human CD271positive melanoma stem cells associated with metastasis establish tumor heterogeneity and long-term growth. Cancer Res 2011;71:3098-109.

17. Krygier S, Djakiew D. Neurotrophin receptor p75(NTR) suppresses growth and nerve growth factor-mediated metastasis of human prostate cancer cells. Int J Cancer 2002;98:1-7.

18. Tabassum A, Khwaja F, Djakiew D. The p75(NTR) tumor suppressor induces caspase-mediated apoptosis in bladder tumor cells. Int J Cancer 2003;105:47-52.

19. Epa WR, Markovska K, Barrett GL. The p75

Cite this article as: Huang J, Yu Q, Zhou Y, Chu Y, Jiang F, Zhu X, Zhang J, Wang Q. A positive feedback loop formed by NGFR and FOXP3 contributes to the resistance of non-small cell lung cancer to icotinib. Transl Cancer Res 2020;9(2):10441052. doi: $10.21037 /$ tcr.2019.12.60 neurotrophin receptor enhances TrkA signalling by binding to Shc and augmenting its phosphorylation. J Neurochem 2004;89:344-53.

20. Zheng HC. The molecular mechanisms of chemoresistance in cancers. Oncotarget 2017;8:59950-64.

21. Imai T, Tamai K, Oizumi S, et al. CD271 defines a stem cell-like population in hypopharyngeal cancer. PLoS One 2013;8:e62002.

22. Phi LTH, Sari IN, Yang YG, et al. Cancer stem cells (CSCs) in drug resistance and their therapeutic implications in cancer treatment. Stem Cells Int 2018;2018:5416923.

23. Saida Y, Watanabe S, Tanaka T, et al. Critical roles of chemoresistant effector and regulatory t cells in antitumor immunity after lymphodepleting chemotherapy. J Immunol 2015;195:726-35.

24. Byrne WL, Mills KH, Lederer JA, et al. Targeting regulatory T cells in cancer. Cancer Res 2011;71:6915-20.

25. Frydrychowicz M, Boruczkowski M, Kolecka-Bednarczyk A, et al. The dual role of treg in cancer. Scand J Immunol 2017;86:436-43.

26. Ishibashi Y, Tanaka S, Tajima K, et al. Expression of Foxp3 in non-small cell lung cancer patients is significantly higher in tumor tissues than in normal tissues, especially in tumors smaller than $30 \mathrm{~mm}$. Oncol Rep 2006;15:1315-9.

27. Peng J, Yu Z, Xue L, et al. The effect of foxp3overexpressing Treg cells on non-small cell lung cancer cells. Mol Med Rep 2018;17:5860-8.

28. Triulzi T, Tagliabue E, Balsari A, et al. FOXP3 expression in tumor cells and implications for cancer progression. J Cell Physiol 2013;228:30-5.

29. Dimitrakopoulos FI, Papadaki H, Antonacopoulou AG, et al. Association of FOXP3 expression with non-small cell lung cancer. Anticancer Res 2011;31:1677-83.

30. Fu HY, Li C, Yang W, et al. FOXP3 and TLR4 protein expression are correlated in non-small cell lung cancer: Implications for tumor progression and escape. Acta Histochem 2013;115:151-7.

31. Zheng Y, Josefowicz SZ, Kas A, et al. Genome-wide analysis of Foxp3 target genes in developing and mature regulatory T cells. Nature 2007;445:936-40. 